\title{
PROMOVENDO INTERCONEXÕES: CONTRIBUIÇÕES DA DIVISÃO DE QUÍMICA DE MATERIAIS NOS 40 ANOS DA SBQ
}

\author{
Célia Machado Ronconi \\ Instituto de Química, Universidade Federal Fluminense, Campus do Valonguinho, Outeiro São João Batista s/n, Centro, 24020- \\ 150 Niterói - RJ, Brasil \\ Recebido em 01/04/2017; aceito em 04/05/2017

\begin{abstract}
ENABLING INTERCONNECTIONS: CONTRIBUTIONS OF THE MATERIALS CHEMISTRY DIVISION IN 40 YEARS OF THE BRAZILIAN CHEMICAL SOCIETY. Enabling interdisciplinary discussion has been one of the main goals of the Division of Materials Chemistry since its creation in 1993. Over its twenty-four years of existence, the division has disseminated scientific works with great diversity of topics and impacts. The present article seeks to outline the trajectory of the Division of Materials Chemistry since its creation, showing its main indicators of productivity and academic relevance as well as its main challenges.
\end{abstract}

Keywords: Materials Chemistry Division; The Brazilian Chemical Society; $40^{\text {th }}$ Years Anniversary

\section{INTRODUÇÃO}

A Sociedade Brasileira de Química (SBQ) celebra seu aniversário de quarenta anos em 2017. Não obstante essa data tão expressiva, neste ano, pela primeira vez na América do Sul, também ocorrerá o $46^{\circ}$ Congresso Mundial de Química da International Union of Pure and Applied Chemistry (IUPAC). Como parte das comemorações, as diferentes divisões da SBQ foram incumbidas de apresentar a evolução da química brasileira nas suas respectivas áreas de atuação, bem como a inserção destas áreas no contexto internacional.

Incialmente será feita uma exposição do contexto no qual a SBQ e a Divisão de Química de Materiais (DQM) foram criadas. Em seguida será feita a análise da inserção internacional da Química de Materiais. Essa análise foi embasada em relatórios técnicos publicados pela IUPAC, que apontam recomendações com relação a definições e tendências futuras para a área. Foram examinados ainda documentos do National Science Foundation (agência federal americana de fomento à pesquisa), que apontam temas desafiadores para a área de Química de Materiais.

Na tentativa de delinear o perfil da DQM na SBQ foram consultados artigos publicados pelos ex-diretores da divisão, bem como livros de resumos das reuniões anuais e atas. Até o presente momento foram feitas duas análises evolutivas da área de Química de Materiais na SBQ: uma pelo Prof. Oswaldo Luiz Alves (UNICAMP) em $1998,{ }^{1}$ cinco anos após a criação da divisão, e outra feita pelos Professores Adley Forti Rubira (UEM), Aldo José Gorgatti Zarbin (UFPR), Fernando Galembeck (UNICAMP), Oswaldo Luiz Alves (UNICAMP) e Miguel Jafelicci Júnior (UNESP) em 2002, em virtude da comemoração dos vinte e cinco anos de existência da SBQ. ${ }^{2}$ Essa última análise apresentou um quadro evolutivo da DQM no período de 1998 a 2002, com base no número de trabalhos apresentados, participação regional e classificação dos temas (divididos em materiais poliméricos e não-poliméricos). No presente trabalho, contudo, optou-se em organizar os materiais seguindo uma tendência em discussão na IUPAC, de acordo com suas propriedades/funções/ aplicações. $^{3}$

\section{CRIAÇÃO DA SBQ E DA DIVISÃO DE QUÍMICA DE MATERIAIS}

A SBQ foi criada no contexto do Estado Autoritário brasileiro (1964-1985) que tentava restringir as pesquisas científicas no país, com proibições de reuniões científicas e cortes nos orçamentos destinados à pesquisa. ${ }^{4,5} \mathrm{~A}$ missão da SBQ era atender à necessidade de uma organização que vislumbrasse as especificidades da pesquisa na área de química no Brasil. ${ }^{6}$ As reuniões anuais da SBQ ocorriam de forma correlata às reuniões da Sociedade Brasileira para o Progresso da Ciência (SBPC) e passaram a funcionar de maneira totalmente autônoma somente em 1990. ${ }^{7,8}$ Essa desvinculação da SBPC possibilitou ampliar significativamente a pluralidade de temas discutidos em suas reuniões. A partir de 1992, houve uma grande estruturação da SBQ que incluiu a implantação das divisões científicas. ${ }^{9,10}$ Muito embora tenha representado um avanço significativo, pois criou vários nichos para que a comunidade discutisse suas pesquisas primordiais, $\mathrm{o}$ debate interdisciplinar destes nichos e de suas diferentes pluralidades não era fomentado por completo. Diante da diversidade de temas, tornava-se cada vez mais evidente a interconexão e interdisciplinaridade dos trabalhos anualmente discutidos, o que colocava em relevo a necessidade de um mecanismo que sistematizasse e formalizasse tal situação. Dentro dessa complexidade é que se inseriu a criação da Divisão de Química de Materiais em 1993.,2

\section{A QUÍMICA DE MATERIAIS NA ESTRUTURA DA IUPAC}

Apesar de a disciplina "Química de Materiais" ter surgido na década de $1970,{ }^{2}$ somente em 2005 teve início um projeto da International Union of Pure and Applied Chemistry (IUPAC), liderado pelo Prof. Peter Days da Universidade de Londres, intitulado “O que é Química de Materiais?”, cujo objetivo era aprovar uma definição internacionalmente aceita para esta disciplina e discutir sua representação dentro da estrutura da IUPAC. ${ }^{11}$

Atualmente, as atividades dessa área na IUPAC são supervisionadas pelo Subcomitê Interdivisional de Química de Materiais (SIQM), ${ }^{3}$ que por sua vez faz parte da Divisão de Química Inorgânica. ${ }^{12}$ As publicações geradas a partir do projeto "O que é Química de Materiais?" mostram que esta área foi uma das que mais cresceu na Química Pura e Aplicada, contribuindo com uma fração significativa para as publicações em Ciências Químicas. ${ }^{13,14}$ Tal constatação se

*e-mail: cmronconi@id.uff.br 
fundamentou no número crescente de artigos científicos submetidos, além das citações de artigos publicados em periódicos dedicados total ou parcialmente à área de Química de Materiais, levando-se em consideração o respectivo fator de impacto (FI) de cada um dos periódicos, evidentemente. ${ }^{15}$ É importante ressaltar que após o período avaliado pelo SIQM da IUPAC (até 2009), a área continua em plena expansão e, como reflexo, vários periódicos novos foram lançados para atender à sua crescente demanda.

Os periódicos mais conhecidos na área de Química de Materiais com seus respectivos fatores de impacto (2016 Journal Citation Reports) em ordem decrescente são: Nature Materials (FI = 38,891, Nature Publishing Group - NPG), Advanced Materials (FI = 18,960, Wiley-VCH), Advanced Functional Materials $(\mathrm{FI}=$ 11,380, Wiley-VCH), Materials Today (FI = 10,793, Elsevier) e Chemistry of Materials (FI = 9,407, American Chemical Society - ACS). Recentemente foram lançados periódicos novos, como Materials Horizon (2014, FI =9,095, Royal Society of Chemistry - RSC), Materials Chemistry Frontiers (2015, RSC), Materials Today Chemistry (2016, Elsevier) e Applied Materials Today (2015, Elsevier).

Objetivando contemplar a diversidade de temas da área de Química de Materiais, três novos periódicos surgiram em 2013, em substituição ao tradicional o Journal of Materials Chemistry (RSC), lançado originalmente em 1991: o Journal of Materials Chemistry $A$ (materiais para produção e conversão de energia, materiais sustentáveis, etc. $\mathrm{FI}=8,262$ ), $B$ (materiais para biomedicina, cuidados médicos, materiais biomiméticos, etc. FI $=4,872$ ) e $C$ (materiais para armazenamento de informação, materiais ópticos, magnéticos, etc. $\mathrm{FI}=5,066$ ).

Outros periódicos importantes são: Nano Letters $(\mathrm{FI}=13,779$, ACS), ACS Nano (FI = 13,334, ACS), Biomaterials ( $\mathrm{FI}=8,387$, Elsevier), Small (FI = 8,320, Wiley-VCH), Nanosacale (FI = 7,760, RSC), Applied Materials and Interfaces (FI = 7,145, ACS), Soft Matter (FI = 3,798, RSC), ACS Biomaterials Science \& Engineering (2015, ACS). As revistas voltadas para as áreas de físico-química, inorgânica, macromoléculas e química de superfícies têm uma percentagem grande dos seus artigos classificados como "Química de Materiais", por exemplo, o Journal of Physical Chemistry B (FI $=3,187), C(\mathrm{FI}=4,509)$ e o Journal of Physical Chemistry Letters $(\mathrm{FI}=8,539)$ da ACS.

Reconhecida a importância e o crescimento da área de Química de Materiais dentro das Ciências Químicas, o projeto coloca em discussão a possibilidade de criação da Divisão de Química de Materiais dentro da IUPAC, que hoje conta com oito divisões: I. Physical and Biophysical Chemistry; II. Inorganic Chemistry; III. Organic and Biomolecular Chemistry; IV. Polymer; V. Analytical Chemistry; VI. Chemistry and the Environment; VII. Chemistry and Human Health e VIII. Chemical Nomenclature and Structure Representation. ${ }^{16}$ Além da proposta de inserção da Química de Materiais como uma nova divisão dentro da estrutura da IUPAC, o projeto produziu uma definição sobre o que faz e o que não faz a Química de Materiais. ${ }^{13,14}$

\section{Definição de Química de Materiais}

A seguinte definição de Química de Materiais é recomendada pela IUPAC: "A Química de Materiais consiste na aplicação da química no planejamento, síntese, caracterização, processamento, entendimento e utilização de materiais, particularmente aqueles com propriedades físicas úteis ou potencialmente úteis”. ${ }^{13,14}$ Esta definição está esquematicamente ilustrada na Figura 1.

A Química de Materiais busca o entendimento de como átomos, íons ou moléculas estão arranjados num sólido ou líquido organizado, e.g. cristal líquido, e como esta disposição determina suas

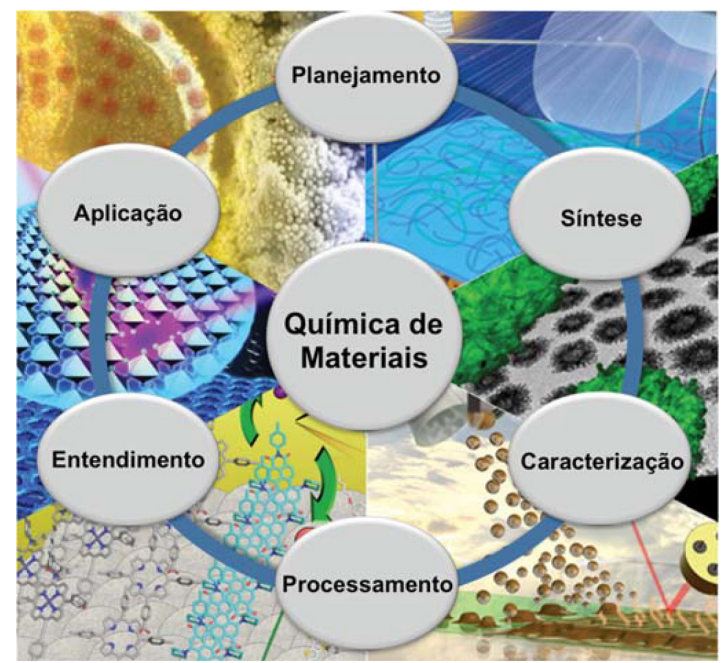

Figura 1. Ilustração representando a definição da Química de Materiais. As figuras foram reproduzidas com autorização das capas do J. Mater. Chem. 2012, 22, $n^{\circ}$ 46-48; J. Mater. Chem. A 2017, 5, $n^{\circ} 11-12$ e J. Mater. Chem. A 2017, 28, 12

propriedades e, consequentemente, suas aplicações. ${ }^{17}$ A síntese de novas substâncias químicas em escala nano, micro ou macroscópica não deve ser entendida como Química de Materiais e sim, como Síntese Química. ${ }^{13,14}$ No caso de um nanomaterial, suas propriedades e aplicações devem derivar, necessariamente, de seu tamanho nanométrico ou de sua elevada razão superfície/volume. ${ }^{18}$ Portanto, na Química de Materiais o estudo deve envolver a relação entre estrutura/ propriedade e a indicação da aplicação do material. O entendimento da composição química de novos sólidos, das interações presentes e das propriedades advindas é parte da Ciência Química.

\section{A EVOLUÇÃO DA QUÍMICA DE MATERIAIS NO CONTEXTO MUNDIAL}

Existem registros históricos que mostram que a humanidade faz uso de materiais há pelo menos 10.000 anos. ${ }^{19}$ Inicialmente, o homem utilizava os materiais encontrados na natureza (pedra, madeira, metal) sem praticamente alterá-los. Posteriormente, o homem aprendeu a preparar alguns materiais, como por exemplo o vidro, empregando calor e produtos químicos. ${ }^{20}$ Aprendeu, também a separar metais de minérios com aquecimento e processos de redução. Na Tabela S1 (Material Suplementar) é mostrada a evolução dos materiais na história da humanidade.

Com a Segunda Guerra Mundial, o crescimento da área é acelerado e, nos anos 1950, são fabricados os transistores de silício, revolucionando a indústria eletrônica (o termo transistor vem do inglês transfer resistor, que significa resistência de transferência ${ }^{21}$ ). Neste mesmo período, os polímeros sintéticos contribuem para o avanço da ciência e tecnologia de materiais, prosseguindo até os dias atuais. No final dos anos 1960 e início dos anos 1970 surge a disciplina "Química de Materiais" devido ao interesse crescente nas propriedades de compostos moleculares e inorgânicos no estado sólido. Na década de 1970 são descobertos os polímeros condutores e os cristais líquidos passam a ser empregados em mostradores de relógios de pulso. $\mathrm{Na}$ década de 1980, o desenvolvimento de supercondutores em altas temperaturas coloca em foco os óxidos metálicos. A descoberta dos nanotubos de carbono no início da década de 1990 e de suas propriedades mecânicas, elétricas e térmicas excelentes vislumbraram novas aplicações, como por exemplo, a incorporação de nanotubos em materiais convencionais, como polímeros ou polímeros condutores, para melhorar as propriedades mecânicas e elétricas dos compósitos 
resultantes. Os nanotubos de carbono são extensamente investigados e têm aplicações em dispositivos nano-eletrônicos, dispositivos de

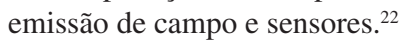

O século XXI inicia com a promessa de substituição dos circuitos integrados de silício pela eletrônica molecular. ${ }^{23}$ Em 1965, Gordon Moore, um dos cofundadores da Intel, fez uma previsão para a velocidade de crescimento da tecnologia baseada em silício, que ficou conhecida como Lei de Moore. ${ }^{24}$ Essa lei é obedecida pela indústria eletrônica e uma de suas consequências é o aumento na capacidade de memória dos processadores. Contudo, a fabricação de computadores cada vez mais rápidos enfrenta uma série de limitações físicas relacionadas ao material empregado na construção dos dispositivos, bem como nas técnicas de litografia aplicadas para criar os circuitos integrados sobre wafer de silício.

A eletrônica molecular surge como uma alternativa para contornar essas limitações, pois um número muito maior de moléculas poderia ser integrado em um circuito. Em 2000, os grupos de pesquisa dos Professores James Fraser Stoddart (Northwestern University, EUA) e James Heath (California Institute of Technology, Caltech) prepararam dispositivos de memória empregando moléculas chamadas catenanos $($ do Latim, catena $=$ cadeia $)$, onde o movimento coletivo destas moléculas no estado sólido alternaria entre o estado ligado (on) e desligado (off). Essas moléculas são constituídas por dois macrociclos mecanicamente entrelaçados que se movimentam entre dois sítios de reconhecimento quando um estímulo elétrico é aplicado. Medidas elétricas mostraram que os dispositivos apresentam um estado de alta (on) e baixa (off) condutividade, portanto, as moléculas atuam como chaveadores moleculares no estado sólido, Figura 1S (Material Suplementar). O conceito fundamental envolvido no funcionamento do dispositivo molecular fabricado por Stoddart e Heath é o da coletividade. Esse conceito vem sendo cada vez mais investigado em Química de Materiais e é considerado pelo National Science Foundation (NSF), agência federal americana de fomento à pesquisa científica, um dos temas de pesquisa a ser explorado mais difícil e desafiador na área de Química de Materiais e de Nanotecnologia. ${ }^{25}$

Em 2004, o grafeno ganha destaque após os Professores Andre Geim e Kostya Novoselov, da Universidade de Manchester, mostrarem que esse material, constituído por uma monocamada de grafite, era transparente, estável em condições ambientes e com alta condutividade e mobilidade elétricas. ${ }^{26} \mathrm{~A}$ partir de então, esse material passou a ser amplamente investigado e novas propriedades foram descobertas, como a elevada resistência mecânica (200 vezes maior que do aço), flexibilidade, leveza, impermeabilidade entre outras. Com tantas propriedades interessantes, o grafeno é um do materiais mais investigados atualmente e tem aplicações em eletrônica, spintrônica, fotônica, sensores, células fotovoltaicas, células a combustível, no armazenamento de hidrogênio, etc. ${ }^{27}$ Um desafio nessa área é produzí-lo com qualidade em escala industrial.

Os materiais híbridos orgânicos-inorgânicos multifuncionais, incluindo os compósitos, são uma classe imensa de materiais que vem sendo investigada desde a segunda metade do século $\mathrm{XX}$. Recentemente, contudo, alguns desses materiais, como as redes de coordenação ou redes metal-orgânicas (Metal Organic Frameworks, cujo acrônimo é MOFs) têm sido sintetizadas "sob medida" de maneira a apresentarem propriedades específicas. O Prof. Omar Yaghi (Universidade da Califórnia, Berkely) tem obtido redes de coordenação com valores de área de superfície específica de até $8000 \mathrm{~m}^{2} \mathrm{~g}^{-1}{ }^{28}$ Em parceria com a empresa química alemã BASF, esses materiais estão sendo empregados para descontaminar água, em tanques de combustível para armazenar hidrogênio e na captura de $\mathrm{CO}_{2}{ }^{29}$

Merece destaque ainda a área de biomateriais - naturais e sintéticos - para aplicações médicas, como próteses, reparo de retinas descoladas e dispositivos cardiovasculares. ${ }^{30,31}$

\section{A DIVISÃO DE QUÍMICA DE MATERIAIS (DQM) NOS 40 ANOS DA SBQ}

Desde a sua criação na SBQ em 1993, a DQM vem atuando na fronteira de diversas áreas, agrupando estudantes e profissionais de Química (Inorgânica, Físico-Química, Orgânica e Analítica), de Engenharia de Materiais, de Engenharia Química, de Física entre outras áreas.

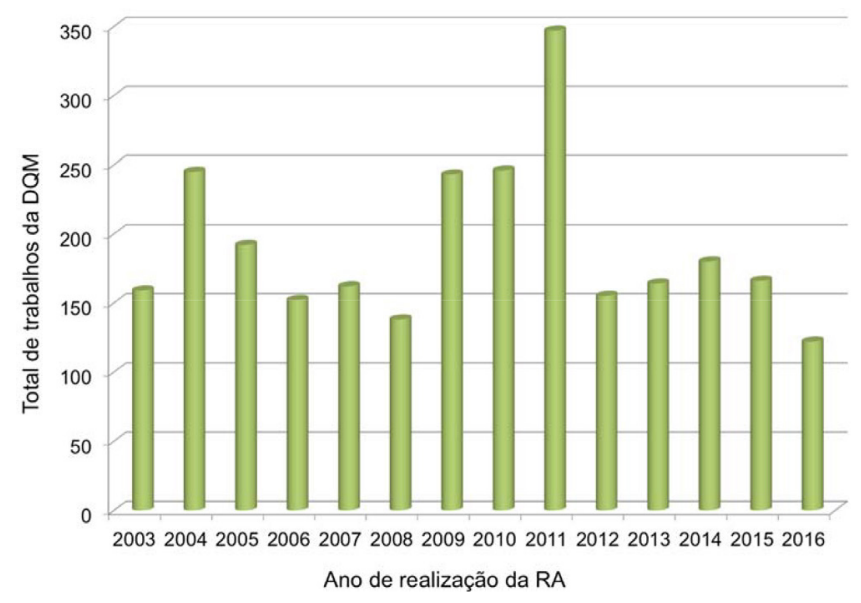

Figura 2. Total de trabalhos da DQM apresentados nas RAs da SBQ de 2003 a 2016 (média $=190$ trabalhos)

O objetivo da DQM é subsidiar debates, descobertas, inovação, colaborações e intercâmbios nos mais variados ramos da Química de Materiais. Para alcançar esses objetivos, a DQM participa ativamente das atividades das reuniões anuais da SBQ convidando palestrantes nacionais e internacionais, oferecendo workshops, minicursos, sessões temáticas, sessões coordenadas, sessões de painéis e prêmios. A programação da DQM é organizada pela diretoria da divisão com a cooperação dos filiados, que são frequentemente consultados - o canal de comunicação é por e-mail, página eletrônica (http://www. sbq.org.br/materiais/) e página no facebook (https://www.facebook. com/MATSBQ) - e incentivados a contribuírem com sugestões que atendam as contingências da comunidade científica.

Devido à sua natureza multidisciplinar, o número de trabalhos apresentados na DQM é bastante expressivo, evidenciando que é uma divisão consolidada dentro da SBQ. Nas Reuniões Anuais (RAs) de 1993 a 2002, o número médio de trabalhos foi de $121 .{ }^{2}$ Esse número aumentou para 190 nas RAs de 2003 a 2016 (Figura 2), que corresponde a um aumento de $57 \%$. Em 2011, na 34 RA da SBQ em Florianópolis-SC, houve um recorde no número de trabalhos apresentados em todas as divisões, sendo a DQM a quinta divisão com o maior número de trabalhos (347). Já em 2015, a DQM contribuiu com o maior número de trabalhos (166) na 38 RA da SBQ em Águas de Lindóia-SP. ${ }^{32}$ Em 2016, a DQM apresentou 122 trabalhos na 39a RA da SBQ em Goiânia-GO, ficando atrás apenas da tradicional Divisão de Química Orgânica, com 135 trabalhos. ${ }^{33}$ A DQM contribui com cerca de $10 \%$ do total de trabalhos apresentados nas RAs da SBQ. Esse percentual é essencialmente o mesmo relatado no levantamento de 2002. ${ }^{2}$ Portanto, o aumento no número de trabalhos apresentados na DQM nas RAs de 2003 a 2016 é reflexo do aumento no número de participantes nas RAs da SBQ.

O número de filiados à DQM no período de 2003 a 2016 está representado na Figura 3. Esse número é próximo de duzentos, com valor médio de 194. Não há grandes variações no número, indicando que não há evasão e nem inserção de novos filiados.

Como mencionado anteriormente, os temas dos trabalhos 


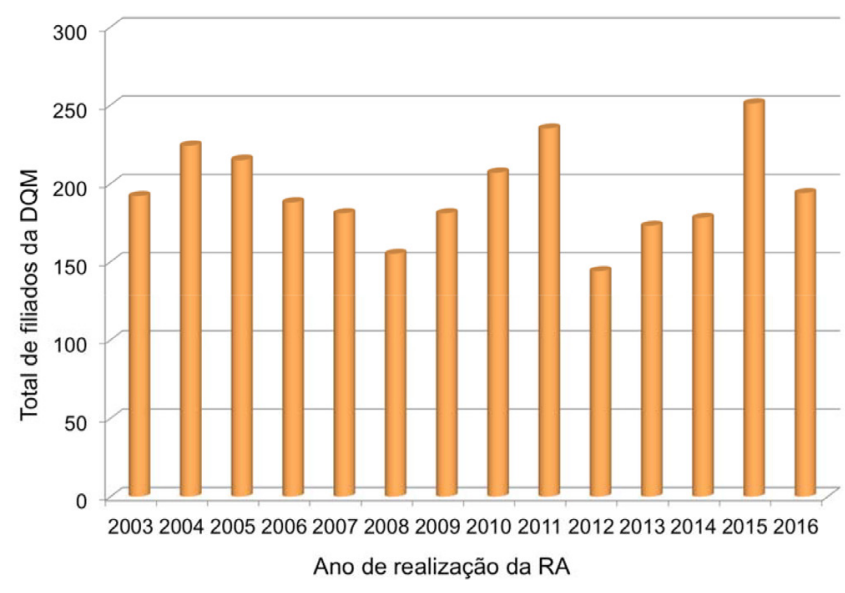

Figura 3. Total de filiados à DQM de 2003 a 2016 (média = 194 filiados)

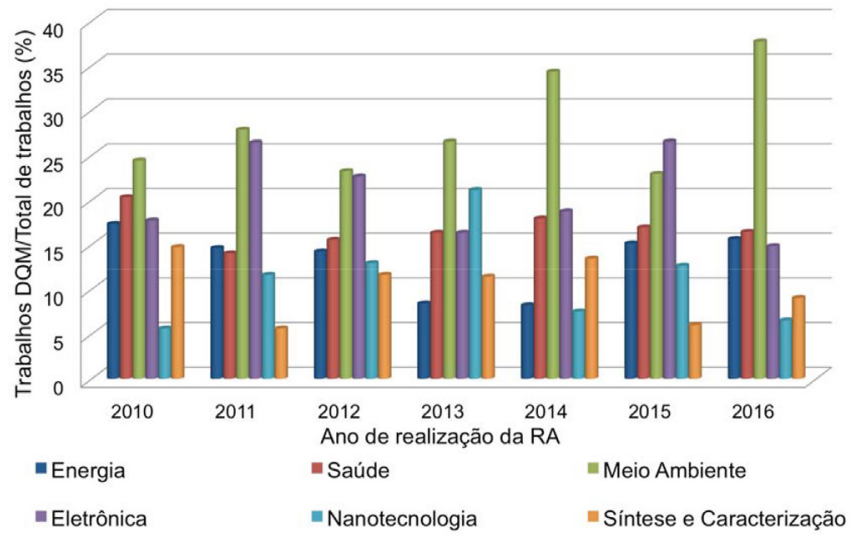

Figura 4. Distribuição dos trabalhos da DQM por temas

apresentados na DQM foram organizados por propriedades/funções/ aplicações, conforme a proposta em discussão no SIQM da IUPAC. ${ }^{34}$ Foram considerados os seguintes temas para a análise do perfil: materiais para energia, saúde, eletrônica, nanotecnologia e meio ambiente. ${ }^{35}$ Para cada área de aplicação existem diversas subáreas que estão listadas na Tabela 1. Essa análise foi feita para as RAs de 2010 a 2016.

Na Figura 4 está representado o resultado do levantamento dos resumos apresentados na DQM. O tema de maior ocorrência na DQM nas Reuniões Anuais (RAs) da SBQ de 2010 a 2016 (Figura 4 e Tabela 1) envolve a síntese de materiais para aplicações ambientais. Os trabalhos nessa área incluem fotocatalisadores para degradação de corantes, materiais adsorventes para remoção de metais pesados em água, sensores para detecção de metais pesados e compostos orgânicos em águas (herbicidas, pesticidas, hormônios, etc.), polímeros biodegradáveis, entre outros. Portanto, há uma grande preocupação da comunidade científica brasileira que desenvolve projetos em Química de Materiais em tentar solucionar problemas relacionados à contaminação de águas. Cooperação nessa área está sendo feita entre o Brasil e a Alemanha com financiamento da Coordenação de Aperfeiçoamento de Pessoal de Nível Superior (CAPES) e da Fundação Alexander von Humboldt. Essa parceria resultou num projeto chamado Brazilian-German Frontiers of Science and Technology Symposium (BRAGFOST), que visa reunir jovens pesquisadores brasileiros e alemães, apontados por essas agências como futuros líderes científicos, para trocar experiências, discutir suas pesquisas e estabelecer parcerias em temas estratégicos para os dois países. Em 2016, dois temas do simpósio foram sobre "Sensores Inteligentes" e "Disponibilidade de Água para o Desenvolvimento Sustentável". Em 2015, um dos temas foi sobre "Métodos Efetivos de Sequestro (químico) para Proteção do Meio Ambiente", no qual se discutiu métodos para captura e sequestro de gases causadores do efeito estufa. ${ }^{36}$

O segundo tema que ocorreu com maior frequência são os materiais para aplicação em eletrônica, principalmente semicondutores e materiais com propriedades luminescentes com potencial aplicação em LEDs e OLEDs (Figura 4 e Tabela 1).

Os materiais com aplicação na área da saúde estão à frente dos materiais para aplicação no setor de energia. Como esse último setor envolve o desenvolvimento de materiais para células a combustível, baterias, células fotovoltaicas e catálise, provavelmente, esses trabalhos devem estar distribuídos também nas divisões de eletroquímica, catálise e fotoquímica, o que dificulta a análise da contribuição real da DQM para este setor. Na área de saúde os trabalhos envolvem sistemas de veiculação de fármacos, como por exemplo, polímeros, sílicas mesoporosas e nanopartículas magnéticas, polímeros para geração de tecidos artificiais, marcadores luminescentes, filmes bactericidas, etc.

Finalmente, a área de nanotecnologia cresceu até 2013 contribuindo com $20 \%$ do total de trabalhos da DQM, percentagem que diminui em seguida. A dificuldade maior em classificar os trabalhos nessa área é que muitas vezes os resumos tratam somente da síntese e caracterização de materiais em escala nanométrica, sem determinar ou relacionar suas propriedades com esta escala. Portanto, foi acrescentada na Figura 4 uma coluna extra referente aos resumos que reportaram síntese e caracterização, sem o estudo de propriedades ou indicação de aplicação dos materiais. Essa coluna corresponde a uma média de $10,2 \%$ do total de trabalhos apresentados na DQM. Geralmente são trabalhos que propõem novos métodos de síntese ou melhoram sínteses já descritas na literatura, tanto para materiais em escala micro quanto nanométrica. Nota-se que uma grande parte desses trabalhos são produzidos por alunos no início do mestrado ou doutorado e por alunos de iniciação científica (IC), o que é adequado, já que a IC tem como objetivo introduzir o aluno de graduação na pesquisa científica.

Um dado interessante observado é com relação aos trabalhos sobre grafeno. Apesar de esse material ter sido descoberto em 2004, na RA de 2014 foram apresentados poucos resumos de trabalhos

Tabela 1. Áreas e subáreas de aplicação dos materiais

\begin{tabular}{|c|c|c|c|c|}
\hline Energia & Saúde & Meio ambiente & Eletrônica & Nanotecnologia \\
\hline células a combustível & veiculação de fármacos & materiais verdes & isolantes & plasmônica \\
\hline baterias & engenharia de tecidos & captura de $\mathrm{CO}_{2}$ & semicondutores & fotônica \\
\hline catálise & imageamento & remediação ambiental & condutores & \\
\hline $\begin{array}{l}\text { armazenamento de } \\
\text { hidrogênio }\end{array}$ & recobrimentos bactericidas & sensores & $\begin{array}{c}\text { materiais para armazenamento } \\
\text { de informações }\end{array}$ & \\
\hline \multirow[t]{2}{*}{ células fotovoltaicas } & & & $\begin{array}{c}\text { OLEDs (organic light-emitting } \\
\text { diodes) }\end{array}$ & \\
\hline & & & LEDs (light-emitting diodes) & \\
\hline
\end{tabular}


com pesquisa em grafeno, sendo que nas RAs seguintes houve um aumento significativo. Destacam-se os grupos de pesquisa dos Estados do Paraná, Minas Gerais e São Paulo que vem realizando pesquisas importantes sobre grafeno.

Os percentuais de participação das regiões do Brasil nas RAs de 2010 a 2016 são mostrados na Figura 5.

Os resultados mostram que a região Sudeste concentra $64,9 \%$ em média dos trabalhos apresentados na DQM, seguida pela região Sul com 12,8 \%, Nordeste com 10,5\%, Centro-Oeste com 5,8\% e Norte com $0,4 \%$. A produção inter-regional contribui com $9,7 \%$.

Nos últimos anos houve um aumento no percentual de trabalhos realizados em colaboração entre instituições de regiões diferentes. Contudo, a distribuição discrepante dos trabalhos da DQM pelas regiões do Brasil reflete, infelizmente, a própria dinâmica da produção do conhecimento científico nacional, concentrada nos locais de maior adensamento populacional e de investimentos. Esses resultados foram bastante semelhantes aos resultados do levantamento feito em 2002, que mostrou que o Estado de São Paulo concentra a maior parte dos trabalhos apresentados com destaque para grupos de pesquisa localizados nas cidades de Campinas, Araraquara e São Carlos. A realização de RAs itinerantes pela SBQ nos últimos anos, como as de 2011 em Florianópolis-SC, 2014 em Natal-RN e 2016 em Goiânia-GO, pode consistir num instrumento que reduza essa evidente desproporcionalidade.

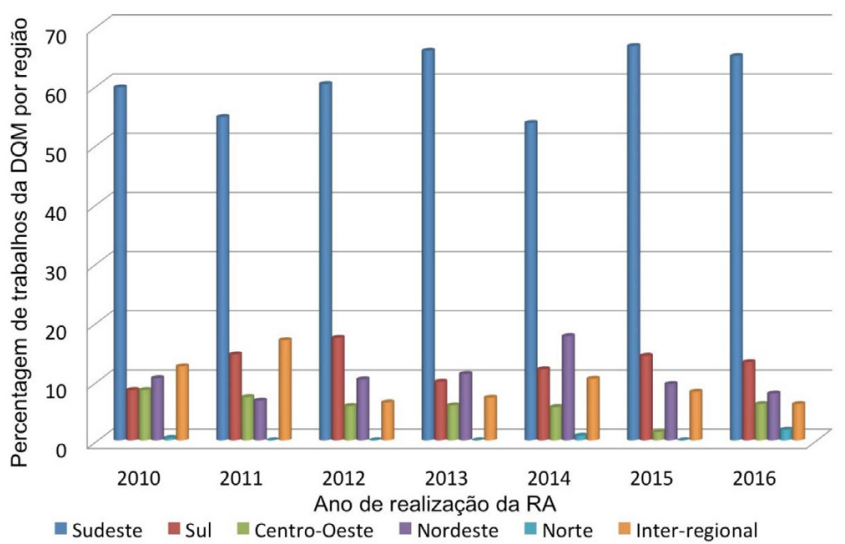

Figura 5. Percentuais de participação regional

O levantamento revelou que apoio da iniciativa privada no desenvolvimento dos trabalhos é ínfimo, o que causa estranheza, haja vista que área de Química de Materiais tem grande apelo tecnológico.

Finalmente, uma pesquisa na base de dados Web of Science, no período de 2012 a 2017, empregando-se dois rótulos de campo combinados pelo operador booleano "and" (Brazil e os periódicos mais importantes na área de Química de Materiais), resultou nos seguintes registros: 119 artigos publicados no periódico Journal of Materials Chemistry (incluindo o Journal of Materials Chemistry A, B e C); 62 no Nanoscale; 49 no Nano Letters; 26 no ACS Nano; 22 no Chemistry of Materials; 14 no Advanced Materials e 03 no Nature Materials. A quantidade de publicações em periódicos dessa magnitude revela a qualidade e diversidade dos trabalhos constantemente produzidos pela Química de Materiais no Brasil.

\section{COMENTÁRIOS FINAIS}

Nestes quarenta anos de existência da SBQ e vinte e quatro anos da DQM, a química logrou um salto de qualidade em sua atuação na pesquisa científica no país. A criação de uma divisão que visava organizar e fomentar ainda mais o debate interdisciplinar e suas diferentes possibilidades e de pesquisa tem se mostrado, ano após ano, uma decisão acertada, uma vez que são cada vez mais significativos os trabalhos que se estabelecem dentro dessa premissa.

Ainda que os propósitos iniciais pareçam ter sido atingidos (e de fato foram, quando consideramos a exclusivamente os indicadores de produtividade e impacto acadêmico) há, entretanto, muito que se aprimorar ainda. A disseminação da pesquisa para além dos centros tradicionalmente estabelecidos dentro da realidade regional brasileira se coloca como um dos desafios mais sensíveis da Divisão de Química de Materiais. Não menos importante é o reconhecimento da necessidade de se ampliar a divulgação dos resultados das pesquisas em Química de Materiais para além do universo acadêmico, uma vez que o investimento proveniente da iniciativa privada é radicalmente tímido. Tal setor deve ter sempre em conta que a inovação é a marca indelével da Química de Materiais.

\section{MATERIAL SUPLEMENTAR}

Material suplementar, contendo uma Tabela referente à história dos materiais e uma Figura representado o funcionamento de um chaveador molecular, está disponível em http://quimicanova.sbq.org. br, na forma de arquivo PDF, com acesso livre.

\section{REFERÊNCIAS}

1. Alves, O. L.; Quim. Nova 1998, 21, 807.

2. Rubira, A. F.; Zarbin, A. J. G.; Galembeck, F.; Alves, O. L.; Jafelicci, M.; Quim. Nova 2002, 25, 75.

3. https://iupac.org/who-we-are/divisions/division-details/?body_ code $=205$, acessada em Fevereiro 2017.

4. Peixoto, M. A.; Quim. Nova 1978, 1, 26.

5. Pinto, A. C.; de Andrade, J. B.; Pardini, V. L.; Quim. Nova 1997, $20,3$.

6. Galembeck, F; Quim. Nova 1997, 20, 5.

7. Bechara, E. J. H.; Viertler, H.; Quim. Nova 1997, 20, 63.

8. https://www.youtube.com/watch?v=VLGAnl525qY, acessada em Janeiro 2016.

9. Rocha-Filho, R. C.; Biaggio, S. R.; Bocchi, N.; Quim. Nova 1997, 20, 66.

10. Vieira, P. C.; Barreiro, E. J.; Quim. Nova 2002, 25, 5.

11. IUPAC in Beijing - Division Roundups Part II; Chem. Int. 2006, 28, 13.

12. https://iupac.org/who-we-are/divisions/division-details/?body_ code $=205$, acessada em Fevereiro 2017.

13. Day, P.; Interrante, L. V.; West, A. R.; Pure Appl. Chem. 2009, 81, 1707.

14. Day, P.; Interrante, L. V.; West, A. R.; Chem. Int. 2009, $31,4$.

15. Para o cálculo do Fator de Impacto (FI) veja a referência: Pinto, A. C.; de Andrade, J. B. Quim. Nova 1999, 22, 448.

16. http://sites.nationalacademies.org/PGA/biso/IUPAC/PGA_059754, acessada em Fevereiro 2016.

17. Fahlman, B. D. Em What is Materials Chemistry?; Springer Netherlands: Netherlands, 2007, cap. 1.

18. Zarbin, A. J. G.; Quim. Nova 2007, 30, 1469.

19. http://eprijournal.com/wp-content/uploads/2016/01/1987-Journal-No.-8. pdf, acessada em Março 2017.

20. Alves, O. L.; Gimenez, I. F.; Mazali, I. O.; Cad. Tematicos Quim. Nova Esc. 2001, Fevereiro, 9.

21. https://pt.wikipedia.org/wiki/Trans\%C3\%Adstor, acessada em Março 2017.

22. Meyyappan, M.; Srivastava, D. Em Handbook of Nanoscience, Engineering, and Technology; Goddard III, W. A.; Brenner, D. W.; Lyshevski, S. E.; Iafrate, G. J., eds.; CRC Press Taylor \& Francis Group: Boca Raton, 2007, cap. 5.

23. Collier, C. P.; Mattersteig, G.; Wong, E. W.; Luo, Y.; Beverly, K.; Sampaio, J.; Raymo, F. M.; Stoddart J. F.; Heath, J. R.; Science 2000, 289, 1172 . 
24. Moore, G. E.; Electronics 1965, 38, 114.

25. https://www.nsf.gov/news/overviews/chemistry/chem_q01.jsp, acessada em Março 2017.

26. Novoselov, K. S.; Geim, A. K.; Morozov, S. V.; Jiang, D.; Zhang, Y.; Dubonos, S. V.; Grigorieva, I. V.; Firsov, A. A.; Science 2004, 306, 666.

27. http://www.graphene.manchester.ac.uk/explore/graphene-city/grapheneweek-2015/, acessada em Março 2017.

28. Furukawa, H.; Cordova, K. E.; O'Keeffe, M.; Yaghi, O. M.; Science 2013, 341, 974.

29. https://www.youtube.com/watch?v=z-ZRhLapO2s, acessada em Março 2017.

30. Chang, S.; Nature 2017, 543, 319.
31. Criscione, J. C.; Nat. Biomed. Eng. 2017, 1, 0046.

32. http://www.sbq.org.br/38ra/programacao-completa/sessoes-de-paineis, acessada em Março 2016.

33. http://www.sbq.org.br/39ra/cdrom/listatrabarea.htm, acessada em Março 2016.

34. https://www.iupac.org/cms/wp-content/uploads/2016/12/Minutes-ofthe-Interdivisional Subcommittee-on-Materials-Chemistry-2015.pdf, acessada em Fevereiro 2017.

35. https://iupac.org/materialschemistryedu/, acessada em Março 2017.

36. https://www.humboldt-foundation.de/web/bragfost-2016.html, acessada em Março 2017. 